\title{
Automatic processing in moiré deflectometry by local fringe direction calculation
}

\author{
Héctor Canabal, J. Antonio Quiroga, and Eusebio Bernabeu
}

\begin{abstract}
An algorithm for accurately extracting the local fringe direction is presented. The algorithm estimates, in the neighborhood of $n \times n$ points, the direction of the gradient that points normal to the local fringe direction. The performance of four different derivative kernels is also compared. Since this method is sensitive to noise and variations in background and amplitude, a preprocessing step is used to limit these error sources. The method has been applied to the moiré deflectogram of a spherical and a progressive addition ophthalmic lens, resulting in a map of the refractive power of these lenses. The results are compared with the data obtained with a commercial focimeter. This technique is useful for analyzing the fringe patterns where the fringe direction is variable and must be obtained locally. (C) 1998 Optical Society of America

OCIS codes: $100.2650,120.4120$.
\end{abstract}

\section{Introduction}

Moiré deflectometry is a well-known technique for mapping ray deflections, based on the moiré and Talbot effects. ${ }^{1}$ In most of the research concerned with automatically processing moiré deflectograms the shift in the fringe from its original position is measured. ${ }^{2}$ However, to measure the fringe shift, fringe tracking and identification methods are needed, making complete automatic evaluation of the moiré deflectogram difficult. Also the number of observed fringes restricts the spatial resolution.

Nevertheless in moiré deflectometry there is a direct relationship between the rotation of the fringes in the moiré pattern and the local focal length of the tested object.3,4 Therefore quantitative information about the local focal length of the tested lens can be obtained from the local fringe direction (LFD) of the corresponding moiré deflectogram.

The LFD provides important information in applications in which automatically processing fringe patterns is required, such as interferometry, filtering, fringe tracking, and, as in our case, moiré deflectograms.

An algorithm for estimating the fringe direction

The authors are with Departamento de Óptica, Facultad de Ciencias Físicas, Universidad Complutense de Madrid, Ciudad Universitaria s/n, 28040 Madrid, Spain.

Received 12 December 1997; revised manuscript received 19 March 1998.

0003-6935/98/255894-08\$15.00/0

(C) 1998 Optical Society of America was presented recently by $\mathrm{Yu}$ and coworkers ${ }^{5,6}$ as an approach to image filtering. Although this filter can efficiently suppress noise and has little blurring effect in fringe patterns, it calculates the LFD with 8 or 16 values only, which is an insufficient resolution for moiré deflectometry. One straightforward method for measuring the LFD is to track the fringe position points and locally compute the slope of the line that fits them, although this method shares the problems depicted above for fringe-shift measurements.

On the other hand, viewing the image as a surface (the gray level representing the height), we can propose a new method of measuring the LFD. It is well known that the vector gradient points in the direction of the maximum rate of change. Hence in an ideal fringe pattern the gradient vector must point normal to the LFD.

Moreover these two methods-fringe tracking and gradient direction-are complementary because their combined domain of application covers the entire image. In fringe tracking the fringe is the points of the image at which the intensity has an extreme value (either maximum or minimum) and consequently can obtain only the LFD in this usually small set of points. The gradient-direction method shows exactly the opposite behavior. In the fringe points the gradient vector is zero or has a small value (a fact that is used in many fringe-tracking algorithms to determine the fringe position), and its direction cannot be determined. In the remaining points the gradient has a nonzero value and its direction can be obtained, providing information in a broader range of points. This method provides more 
information in the sense that it expands the concept of fringe from a few extreme-intensity lines to many isointensity (isogray) lines.

The algorithm presented here estimates directly the gradient direction through the calculation of the $x$ and the $y$ derivatives of the image. This calculation is performed by convolution of the fringe pattern with derivative kernels. In this way, choosing an adequate derivative kernel, we could obtain more LFD resolution than in the method proposed in Ref. 5. This technique is useful for the analysis of fringe patterns where the fringe direction is variable and must be locally obtained, as in moiré deflectometry.

Because this method is sensitive to noise (owing to utilization of the derivative operator) and to variable background and amplitude, a normalization process is presented in Section 2. In Section 3 a comparison of the performance of different derivative kernels is realized, proposing a way to discriminate the best kernel for the computation of the LFD. Finally, in Section 4 the algorithm is applied to the mapping of two ophthalmic lenses, and the results are compared with a commercial focimeter.

\section{Normalized Fringe Pattern}

During the process of generation, acquisition, and digitization, the initially assumed sinusoidal intensity distribution is distorted. A general model of the fringe pattern irradiance $I(x, y)$ is given by

$$
I(x, y)=a(x, y)+b(x, y) \cos [\phi(x, y)]+n(x, y),
$$

where $a(x, y)$ is the background illumination, $b(x, y)$ is the fringe amplitude modulation, $\phi(x, y)$ is the phase variation, and $n(x, y)$ is the additive random noise.

Owing to the derivative nature of the gradient calculation, the effect of the random noise is amplified whereas the effects of the background and the amplitude variation are reduced. The random noise alters locally the fringe direction, and it can be filtered out to some extent by any standard filter, such as median, low pass, or spin filters. In our experience, any standard low-pass filter with a kernel size of $1 / 4$ to $1 / 3$ of the minimum fringe period present in the image can be used to filter out the noise without
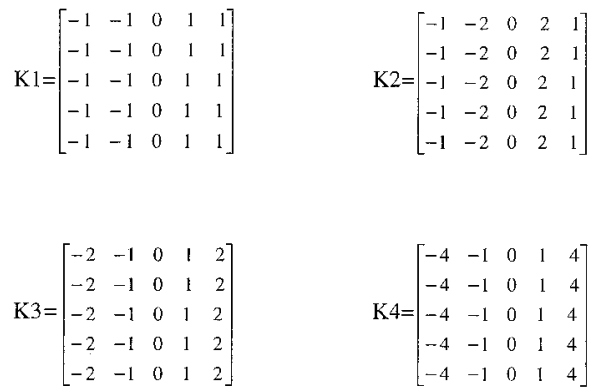

Fig. 1. Tested derivative kernels with a window size of $5 \times 5$ : K1, Prewitt; K2, truncated pyramid; K3, fit to a plane; K4, square weighted. noticeable distortion (from the point of view of the LFD calculation) of the fringe pattern.

Although the effects of the background and the amplitude variations are less noticeable, they must be filtered out for accurate measurements to be obtained. A method for filtering out these errors, based in a two-dimensional (2-D) envelope calculation, is presented in Ref. 7. With this approach a least-squares fitting with a sliding window is used to

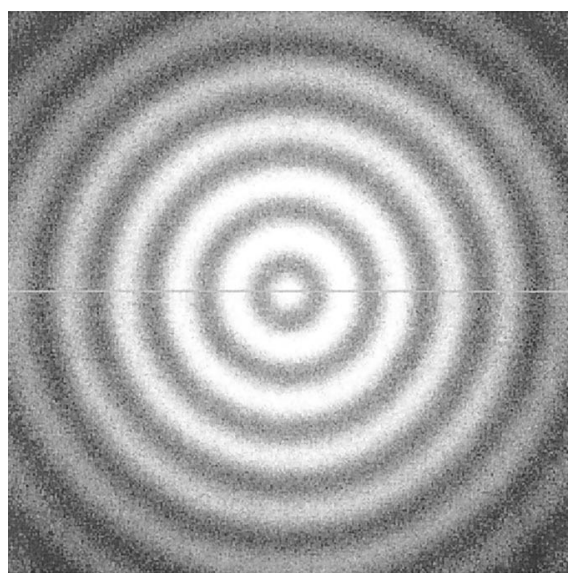

Fig. 2. Simulated circular fringe pattern with a period of 25 pixels with the background and the fringe amplitude Gaussian shaped. A normal random noise of 15 gray levels of amplitude is added.

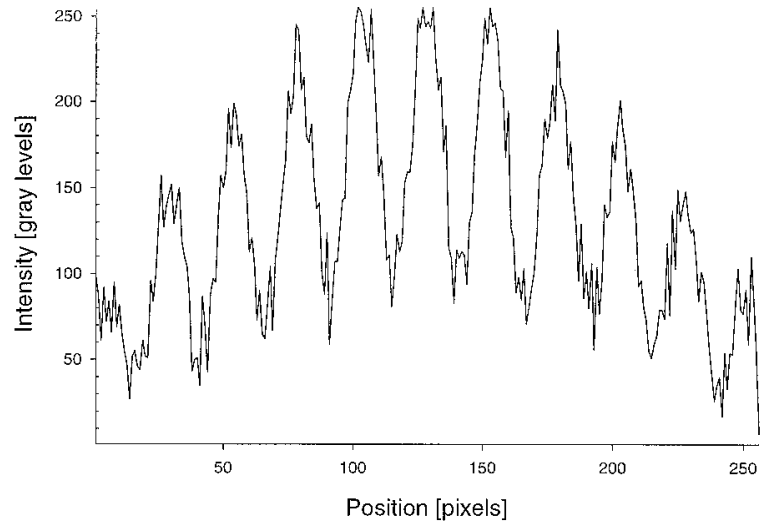

a

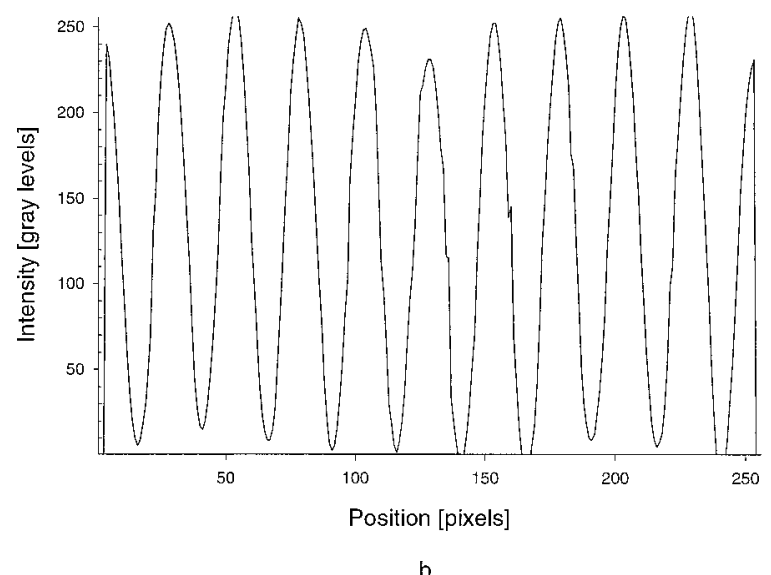

Fig. 3. a, Intensity distributions along the central line of Fig. 2. $\mathrm{b}$, Same line after filtering and fringe normalization. 


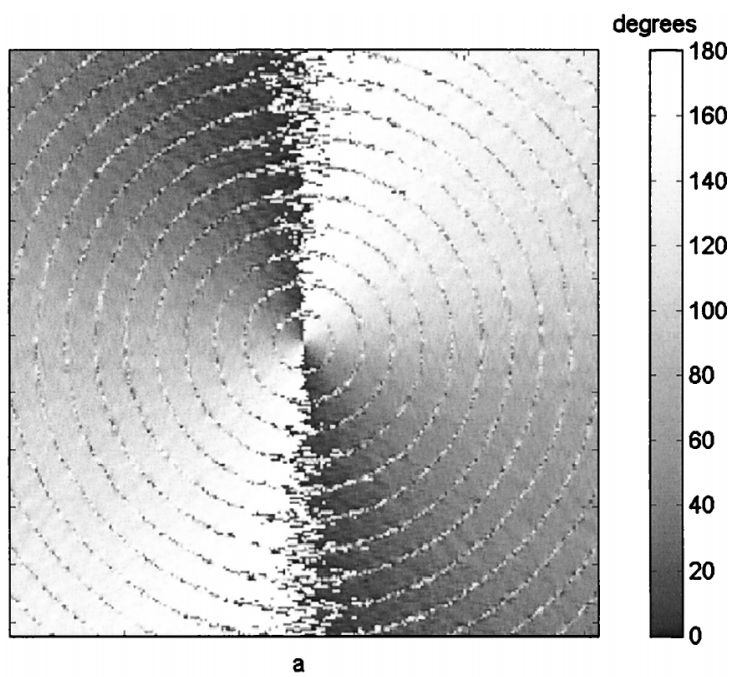

a

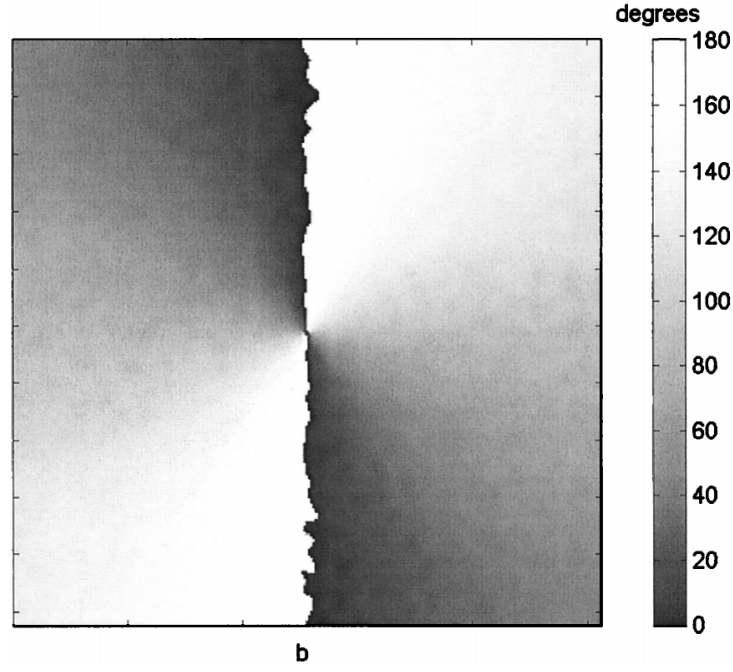

Fig. 4. a, LFD map of Fig. 2. The noisy points correspond to extreme intensity points in the fringe pattern. b, LFD after filtering the low modulation points by interpolation of valid points.

fit the intensity envelopes. For simplicity the extreme gray levels on the fringe center lines are used for fitting. The fitting function proposed in Ref. 7 is a 2-D polynomial with power between 1 and 3 . However, we observed in our setup that a Gaussian fitting function is more adequate and simpler to use. In this case the width, amplitude, and center of the Gaussian are adjusted by a least-squares method.

In this way a preprocessing step was implemented, first, by filtering the high-frequency noise with an adequate kernel size as depicted above, and, second, by computing the 2-D maximum and minimum intensity envelopes by fitting two 2-D Gaussians to the gray levels on bright and dark fringe skeletons, respectively. A filtered fringe pattern without background and amplitude variations, called a normalized fringe pattern, was obtained.

\section{Fringe Simulation and Derivative Kernel Selection}

The gradient of an image $I(x, y)$ at location $(x, y)$ is defined as a 2 -D vector $\mathbf{G}[I(x, y)]=\left[G_{x}, G_{y}\right]=$

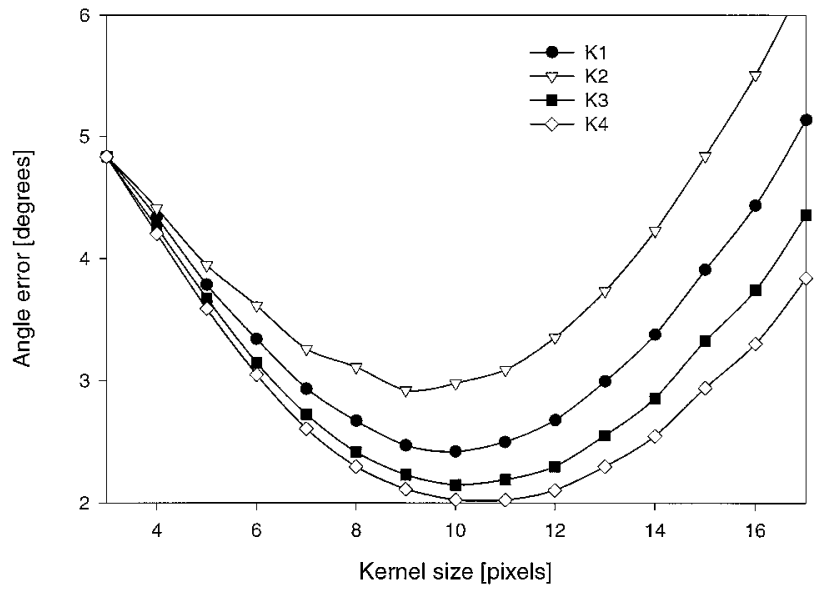

Fig. 5. Fringe angle error versus kernel size for the four derivative kernels depicted in Fig. 1.

$[\partial I / \partial x, \partial I / \partial y]$. The magnitude of this vector is $G(x$, $y)=\left(G_{x}^{2}+G_{y}{ }^{2}\right)^{1 / 2}$, and its angle with respect to the $x$ axis is given by $\alpha(x, y)=a \tan \left(G_{y} / G_{x}\right)$.

We performed a computation of the gradient, estimating the partial derivatives $\partial I / \partial x$ and $\partial I / \partial y$ at every pixel location by differences. Many kernels for computing the gradient components are used commonly for edge detection in images, such as those proposed by Sobel, Roberts, Prewitt, and Frei-Chen. ${ }^{8}$ The performance of four representative kernels, shown in Fig. 1, is analyzed here. K1 is a Prewitt kernel. $\mathrm{K} 2$ is a truncated pyramid, similar to the one proposed by Abdou in Ref. 8. K3 comes from the linear least-squares fit of a plane to the pixels in a rectangular neighborhood, and it gives more importance to the pixels far from the center. ${ }^{9}$ Finally, the $\mathrm{K} 4$ weights are equal to the square of the distance from the center.

These kernels were tested with straight and curved fringes with different angles and also with circular fringes with different periods. Figure 2 shows an example of a simulated fringe pattern of $256 \times 256$ pixels with circular fringes, similar to that used in Refs. 6 and 7. The circular fringes are useful in testing the algorithm because the LFD adopts all the possible values. In Fig. 2 the fringe period is set to 25 pixels, and the background as well as the fringe amplitude is Gaussian shaped. A normal random noise with a standard deviation of 15 gray levels, which represent an error of $\sim 6 \%$, was added. Figures $3 \mathrm{a}$ and $3 \mathrm{~b}$ show the intensity distribution of the central line before and after the normalization step depicted in Section 2.

Figure $4 \mathrm{a}$ is the calculated LFD map. In this image the gray levels represent the fringe direction measured with respect to the horizontal axis, which varies between $0^{\circ}$ and $180^{\circ}$. Note that the gradient magnitude close to a fringe maximum or minimum is near zero, thus producing low modulation points, so the computed gradient direction near these points is usually erroneous and must be discarded. In Fig. 4b the low modulation points were replaced with values 


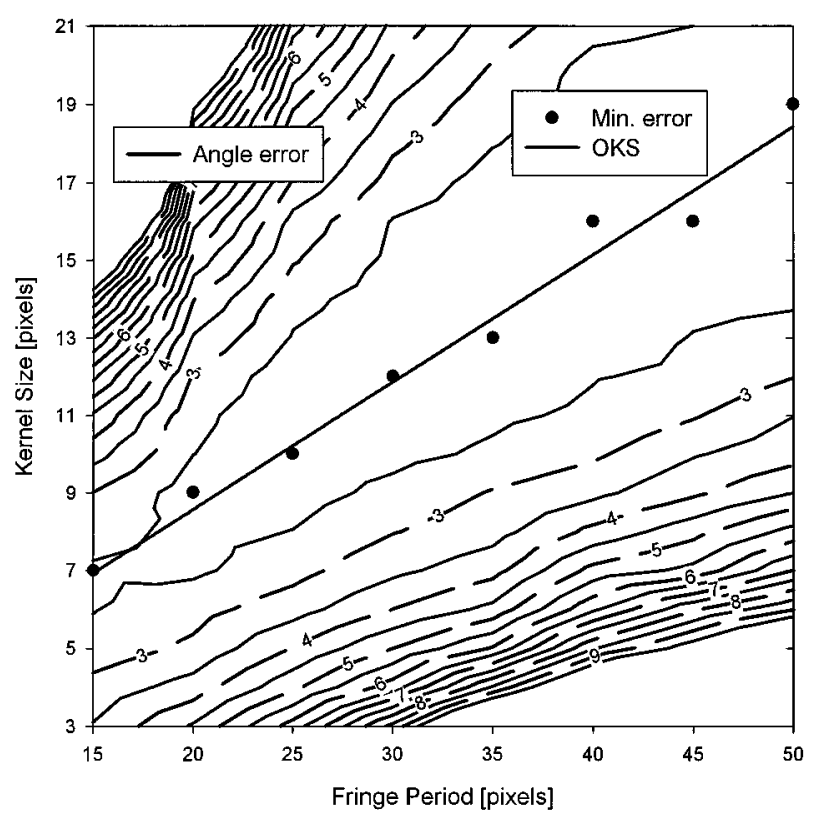

Fig. 6. Contour map of the fringe angle error value with respect to the fringe period and the kernel size. Circular dots are the optimal kernel size for each fringe period. The straight line is the least-squares fitting of these points.

obtained by the inverse distance interpolation in the neighborhood of valid pixels.

If the random noise of the input image is high, it could be propagated to the LFD. If this happens, a filtering of the LFD must be implemented too. In this filtering process care must be taken with the $180^{\circ}$ jumps. An average of the signal in these regions will return incorrect values to the LFD. A way to overcome this problem is filtering this image with a sine-cosine filter, as used commonly for modulus- $2 \pi$ wrapped images. ${ }^{10}$

The calculated LFD is subtracted from the theoretical LFD values for each tested images, and the mean and the standard deviations are calculated. Note that the mean is always around zero, indicating the absence of global systematic errors, while the standard deviation provides a way to estimate the random measurement error. This error depends on several factors, such as noise level, fringe period, fringe curvature, kernel size, and kernel type.

The variation in the error in the function of the kernel size is similar in all the tests performed and for all the kernel types. This behavior for the fringe pattern of Fig. 2 is presented in Fig. 5 for the K1-K4 kernels. Note that the K4 kernel shows the best performance followed by the K3 kernel and that both kernels give more weight to the pixels far from the center.

The kernel size for which the angle error is minimum, i.e., the optimum kernel size (OKS), depends mainly on the fringe period. Figure 6 shows the angle error with respect to the fringe period and the kernel size for the selected kernel K4. This dependence was calculated by using circular fringe patterns like the one depicted in Fig. 2. In this way the

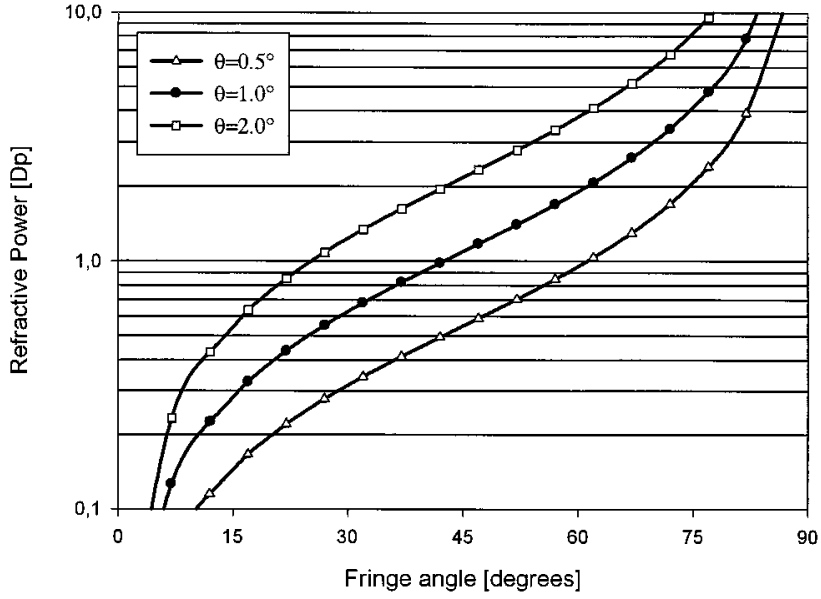

Fig. 7. Refractive power versus fringe angle calculated with grating pitch $p=0.1 \mathrm{~mm}$, separation $z$ is set to the first Talbot plane and for three different angles between the gratings $\theta$.

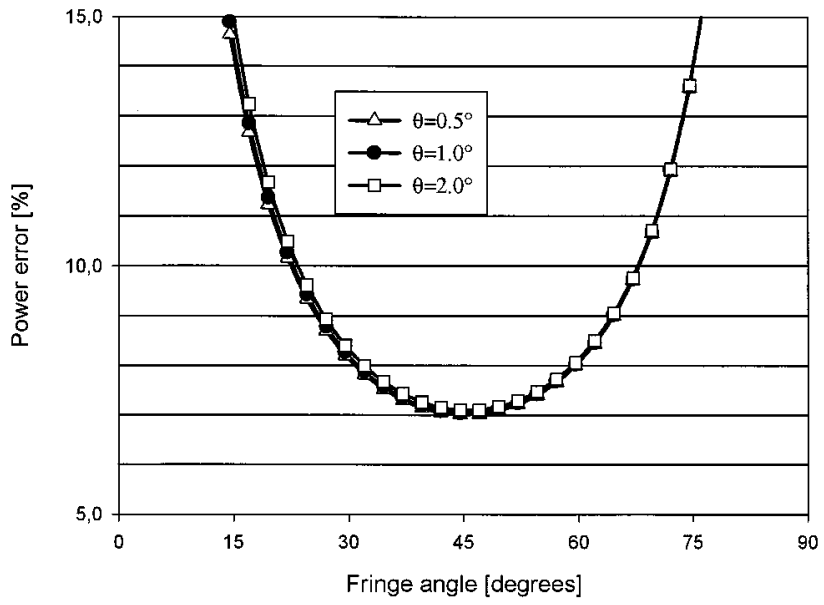

Fig. 8. Relative power error versus fringe angle with the same parameters of Fig. 7 and with $\Delta \alpha=2^{\circ}$. The error is around 7-10\% for $\alpha$ between $20^{\circ}$ and $70^{\circ}$.

K4 curve of Fig. 5 corresponds to a vertical section along column 25 in Fig. 6 and for which the OKS is 10 pixels. A linear least-squares fitting of the position of the OKS versus the fringe period $(F P)$ results in $\mathrm{OKS} \approx 2+\mathrm{FP} / 3$. Hence, for a given fringe pattern, the optimum kernel size with minimal angle error calculation can be estimated based on its average fringe period. However, note that the optimum kernel size depends also on the noise level. The higher the noise level the larger the optimum kernel size, whereas for lower noise levels the optimum kernel size decreases.

\section{Application to Moiré Deflectometry: Characterization of Ophthalmic Lenses}

The experimental setup is a simple moiré deflectometer similar to the one used in Ref. 3 . It consists of an expanded collimated $\mathrm{He}-\mathrm{Ne}$ laser source followed by two Ronchi gratings. The tested lens is placed just before the first grating. The distance between 


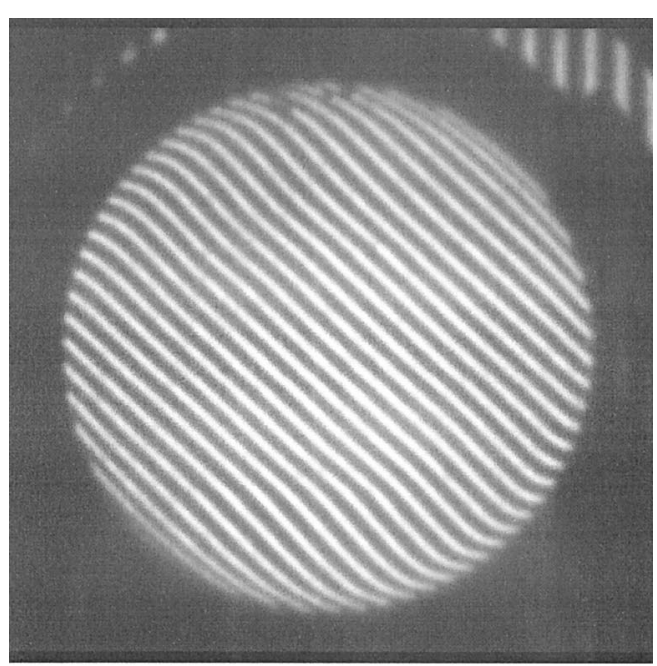

a

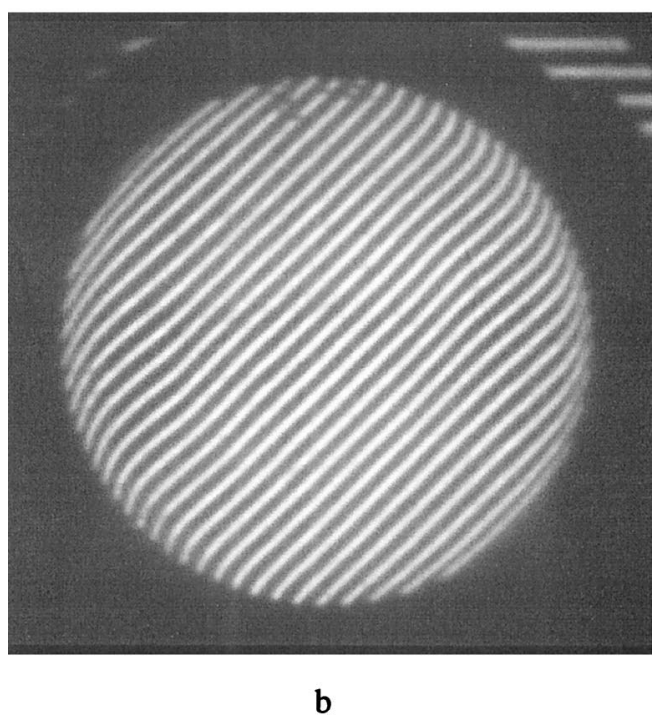

Fig. 9. Deflectograms of a monofocal lens of $+2.00 \mathrm{Dp}$. Note the fringe curve near the lens border and the reference fringes in the upper left corner. The gratings are, a, along the horizontal axis and, $b$, along the vertical axis.

the two gratings is set to a Talbot plane $z=n p^{2} / \lambda$, where $p$ is the grating period $(0.1 \mathrm{~mm}), \lambda$ is the wavelength of the monochromatic source $(632.8 \mathrm{~nm})$, and $n$ is the Talbot plane $(1,2,3, \ldots)$. The angle between the two gratings $\theta$ can be deduced from the fringe period of the reference pattern or can be directly measured. When a lens is placed in the deflectometer, the reference fringe pattern is distorted. The relationship between the fringe angle $\alpha(x, y)$ and the local refractive power of the lens $P(x, y)$ is given by ${ }^{3}$

$$
P(x, y)=\frac{\sin \theta \tan \alpha(x, y)+\cos \theta-1}{z} .
$$

This relationship is presented in Fig. 7 for the first Talbot plane with $p=0.1 \mathrm{~mm}$ and for three different values of $\theta\left(0.5^{\circ}, 1.0^{\circ}\right.$, and $\left.2.0^{\circ}\right)$. The accuracy of this measurement depends on the accuracy of the mea- surement of $\theta, z$, and $\alpha$. In particular, in our setup, the error in the measurement of $\alpha$ represents the main contribution. Figure 8 shows the relative error in $P$ versus the fringe angle $\alpha$, calculated with the same parameters of Fig. 7 and with an angle error of $\Delta \alpha=2^{\circ}$. The optimum measurement zone is obtained by adjusting $\theta$ in such a way that the fringe angle $\alpha$ is around $45^{\circ}$. In this situation, although from Eq. (2), $P=P(\theta)$, the relative error in $P$ is almost independent of the angle between the gratings and can be limited to approximately $7-10 \%$.

Note that in Fig. 7 for a fringe angle of $45^{\circ}$ and for a variation of $\theta$ from $0.5^{\circ}$ to $2^{\circ}$, the value of the refractive power $P$ runs from 0.5 to $2.0 \mathrm{Dp}$ (diopters) while in Fig. 8 the relative error in $P$ is only $7 \%$ for all values of $\theta$. This behavior appears incongruent, but note that we calculated the data of Fig. 8, considering only the error in the measurement of the fringe angle, because the error in $\theta\left(\Delta \theta \approx 0.04^{\circ}\right)$ is negligible in our setup.

Since the deflectometer is sensitive to ray deflec-
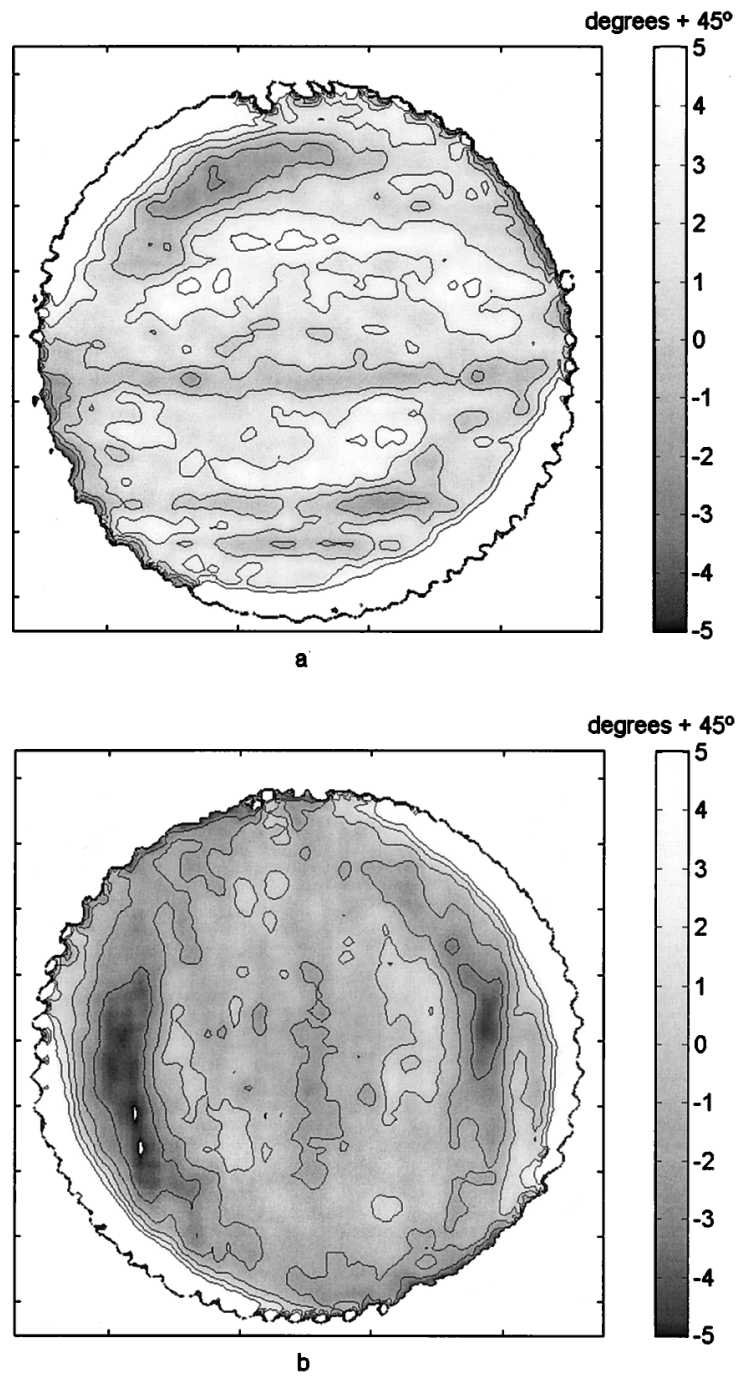

Fig. 10. Map corresponding to the fringe rotation of, a, Fig. 9a and, b, Fig. $9 \mathrm{~b}$. To convey high-contrast information, $45^{\circ}$ has been subtracted from the original fringe rotation maps. 
Dp

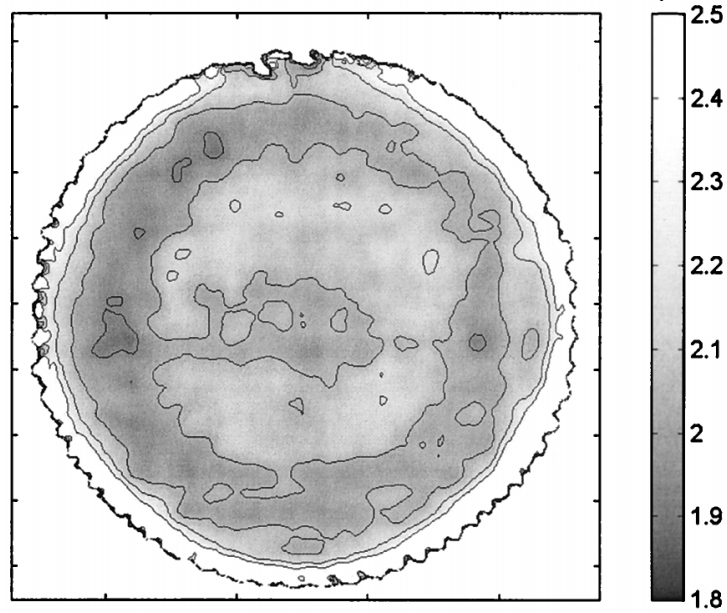

Fig. 11. Local refractive power map for the spherical lens tested.

tions along the axis perpendicular to the grating lines, Eq. (2) gives us only one of the components of the power. To have a complete determination of the refractive power, two images with different grating orientations must be obtained and processed. Also, another two images with reference deflectograms (without lenses) are needed.

The algorithm for acquisition and processing was programmed under Matlab language in a Pentium 166 computer. The complete processing time was $\sim 3 \mathrm{~min}$ for an image size of $512 \times 512$ pixels. To illustrate the performance of our algorithm, we chose two practical examples consisting of a spherical and a progressive addition lens.

Figure $9 \mathrm{a}$ and $9 \mathrm{~b}$ show the deflectograms of a monofocal (spherical) plastic ophthalmic lens with a refractive power of $+2.00 \mathrm{Dp}$, corresponding to the $y$ and the $x$ components of the refractive power. The weak $\mathrm{S}$ shape of the fringes that can be noted near the lens border represents a change in the refractive power due to the spherical aberration. The agreement between the two figures, under a $90^{\circ}$ rotation, verifies that the lens has revolution symmetry.

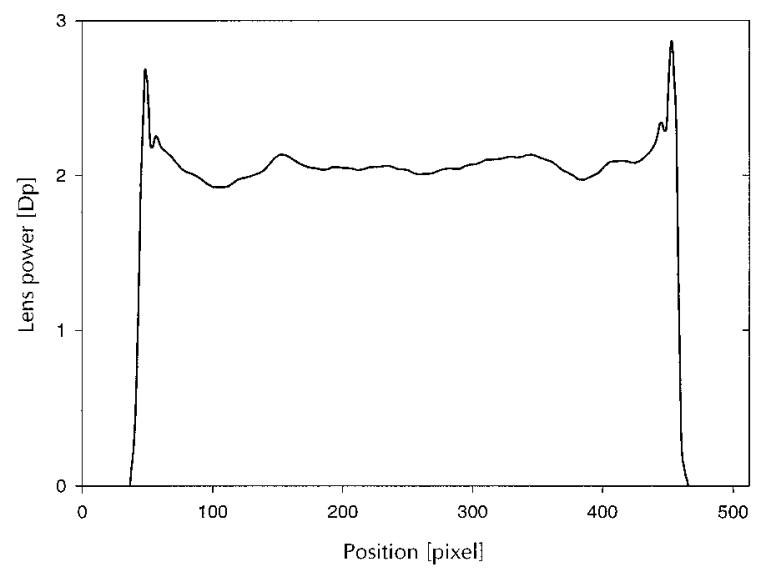

Fig. 12. Profile of the refractive power shown in Fig. 11 along the central horizontal diameter.
As depicted above, the angle between the gratings is adjusted to obtain a fringe angle around $45^{\circ}$ with the lens inserted in the deflectometer. In this case the number of fringes was about 20 for the reference deflectogram, and from it the angle between the gratings was calculated resulting in $\theta_{y}=1.78^{\circ} \pm 0.04^{\circ}$ and $\theta_{x}=1.96^{\circ} \pm 0.04^{\circ}$ for Figs. 9a and $9 \mathrm{~b}$, respectively.

The reference deflectogram must be processed to take into account imperfections in the gratings, that is, the LFD's of the reference deflectogram are subtracted from the LFD's of the deflectogram with a lens. The final fringe rotation maps corresponding to Figs. 9a and $9 \mathrm{~b}$ are shown in Figs. 10a and 10b, respectively. We calculate the local refractive power by using these images and Eq. (2). The composition of the $y$ and the $x$ refractive power data in a map that represents the local power of the lens is shown in Fig. 11. Figure 12 shows a profile along the central horizontal diameter of the lens. As can be seen, the refractive power near the center corresponds to the nominal power of the lens while toward the lens border this value increases owing to spherical aberration.
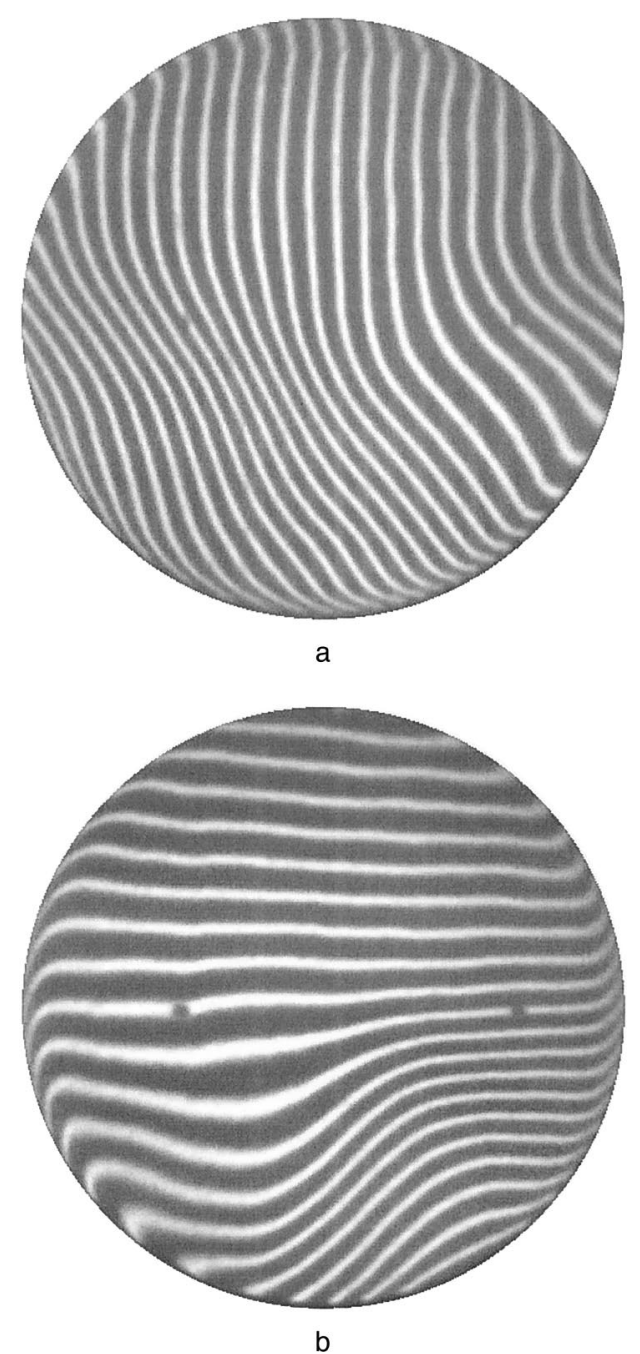

Fig. 13. Deflectograms of a progressive addition lens of $+2.00 \mathrm{Dp}$. The gratings are, a, along the horizontal axis and, b, along the vertical axis. 

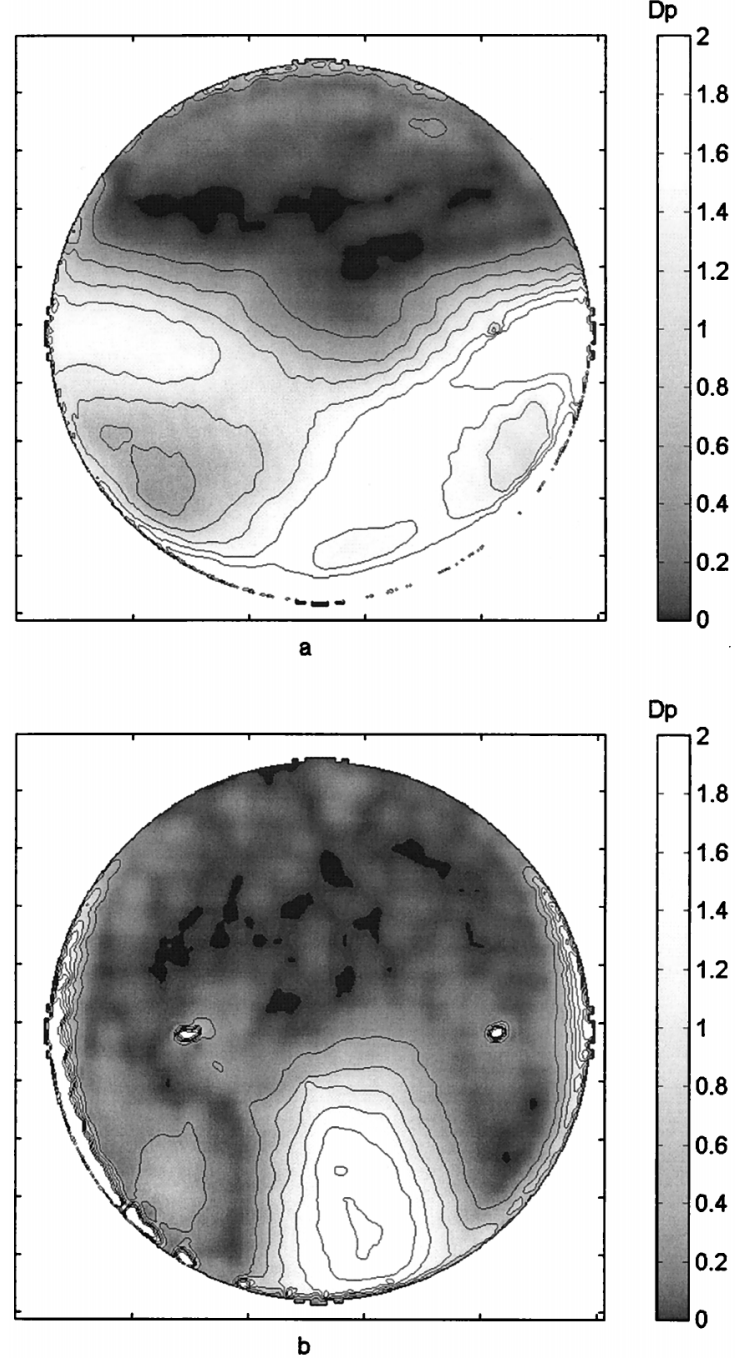

Fig. 14. a, Refractive power map, a, in the $y$ direction and, b, in the $x$ direction, determined from Figs. 12a and 12b, respectively.

The spherical lens is a good conceptual example but a more practical case is the study of a progressive addition lens. Therefore a progressive addition lens with an addition of $+2.00 \mathrm{Dp}$ (Selective from BBGR, Paris) has been tested. The moiré deflectogram with the gratings oriented along the vertical and the horizontal axes are shown in Figs. 13a and 13b, and the components of the refractive power in the $y$ and the $x$ directions are shown in Figs. 14a and 14b. For comparison the same components of the refractive power were measured manually by an automatic focimeter (Humphrey 360) in a rectangular grid of $15 \times$ 15 points. The interpolation of these values in a grid of $64 \times 64$ points is shown in Figs. $15 \mathrm{a}$ and $15 \mathrm{~b}$, presenting a good agreement with our results.

\section{Conclusion}

An algorithm for an accurate extraction of the local fringe direction has been presented. We have shown that the derivative kernels, which give more importance to distant pixels, provide better precision in measurements of the LFD. Also, a simple relation-
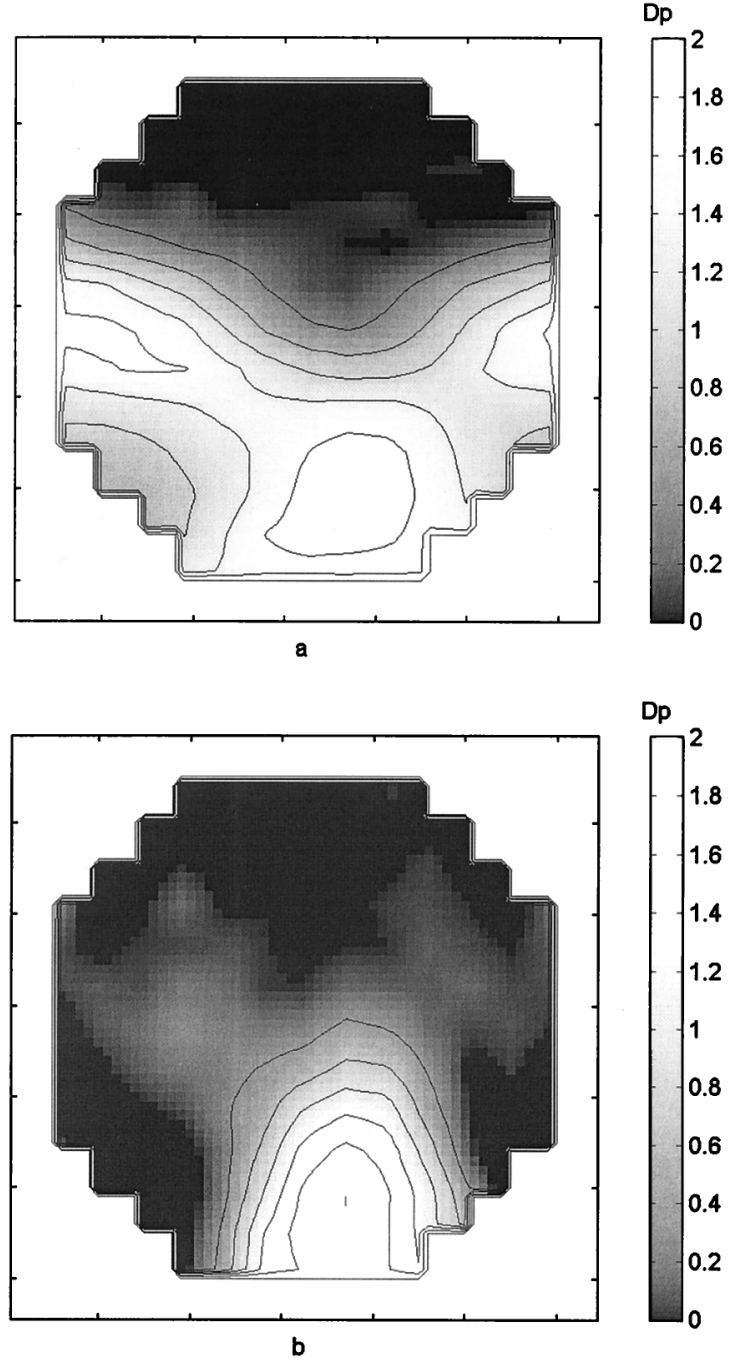

Fig. 15. a, Refractive power map, a, in the $y$ direction and, b, in the $x$ direction, measured with a commercial focimeter.

ship for estimating the optimum kernel size for a fringe pattern with a given fringe period is proposed. The error in the measurement of the fringe angle is around $2^{\circ}$, which corresponds to an error of $7-10 \%$ in the determination of the refractive power. The algorithm has been applied with satisfactory results to the deflectograms of monofocal and progressive ophthalmic lenses.

\section{References}

1. O. Kafri and Y. Glatt, The Physics of Moiré Metrology (Wiley, New York, 1989).

2. M. Servin, R. Rodriguez-Vera, M. Carpio, and A. Morales, "Automatic fringe detection algorithm used for moiré deflectometry," Appl. Opt. 29, 3266-3270 (1990).

3. Y. Nakano and K. Murata, "Talbot interferometry for measuring the focal length of a lens," Appl. Opt. 24, 3162-3166 (1985).

4. Y. Nakano, R. Ohmura, and K. Murata, "Refractive power mapping of progressive power lenses using Talbot interferometry and digital image processing," Opt. Laser Technol. 22, 195-198 (1990).

5. Q. Yu, X. Liu, and K. Andresen, "New spin filters for interfero- 
metric fringe patterns and grating patterns," Appl. Opt. 33, 3705-3711 (1994).

6. Q. Yu and K. Andresen, "Fringe-orientation maps and fringe skeleton extraction by the two-dimensional derivative-sign binary-fringe method," Appl. Opt. 33, 6873-6878 (1994).

7. Q. Yu, K. Andresen, W. Osten, and W. Jueptner, "Noise free normalized fringe patterns and local pixel transforms for strain extraction," Appl. Opt. 20, 3783-3790 (1996).
8. W. Pratt, Digital Image Processing (Wiley, New York, 1991), Chap. 16.

9. H. Vrooman and A. Maas, "Image processing algorithms for the analysis of phase-shifted speckle interference patterns," Appl. Opt. 30, 1636-1641 (1991).

10. B. Ströbel, "Processing of interferometric phase maps as complex-valued phasor images," Appl. Opt. 35, 2192-2198 (1996). 